\title{
A TABULATION OF ORIENTED LINKS
}

\author{
HELMUT DOLL AND JIM HOSTE
}

\begin{abstract}
In this paper we enumerate all prime, nonsplit, oriented, classical links having two or more components and nine or fewer crossings. Our list is complete up to diffeomorphism of $S^{3}$ and complete reorientation of the link. (That is, reorienting every component of the link.) Previously, only tables of nonoriented links have been compiled. Furthermore, we list, in the case of alternating links, all possible minimal diagrams of each link up to orientation. We also include the skein polynomials of each link.

Our methods are direct generalizations of those used by Dowker and Thistlethwaite to enumerate knots. We rely heavily on the HOMFLY and Kauffman polynomials to distinguish inequivalent links. In a few cases these invariants will not suffice and other link invariants are employed. Our table is generated "from scratch" rather than by introducing orientations into already existing nonoriented tables. This provides a check on Conway's table in the range mentioned above.
\end{abstract}

\section{INTRODUCTION}

The history of knot tabulation is a long one, beginning in the last century with Kirkman, Tait, and Little. Assisted by Kirkman, both Tait and Little compiled tables that together presumed to list all knots of ten crossings or less and all alternating knots of 11 crossings [8, 9, 11, 12, 13, 19]. Produced over a period of about 15 years, these tables were truly a remarkable achievement! Of course, Tait and Little could not be absolutely sure that their tables were complete or that they contained no duplications. But with the advent of topology came the development of various knot invariants and so the ability to rigorously distinguish between the knots in the table. Many topologists played a role in this effort, for example Alexander, Reidemeister, and Seifert to name only a few. Tables of knots up to nine crossings were republished in [1] and [17].

Modern knot theorists relied solely on these tables until the 1960's when J. $\mathrm{H}$. Conway described a notation in terms of which it was possible to efficiently list (again by hand but now in only a day!) all knots to 11 crossings and all links to ten crossings [4]. Conway's enumeration provided the first check of the existing tables in the 11-crossing range (Tait and Little had checked their tables against each others) and turned up one duplication and 11 omissions.

Received February 5, 1990; revised October 25, 1990.

1980 Mathematics Subject Classification (1985 Revision). Primary 57M25.

Key words and phrases. Knot, link. 
However, Conway also missed a duplication not caught by Tait and Little, the now famous "Perko pair." These were two entries representing the same tencrossing nonalternating knot and were discovered by the Manhattan lawyer K. Perko [15]. Conway's enumeration of links and his list of nonalternating 11crossing knots were entirely new and could not be checked against any earlier tables.

In the 1970's work still continued to verify that the tables contained no duplications and that they did indeed list only prime knots [16]. The knot tables were extended to 13 crossings by Thistlethwaite and links of 11 crossings were tabulated by A. Caudron [5, 20, 3]. More recently, the discovery of the Jones polynomial and related generalizations has made the task of distinguishing the knots and links in these tables much easier $[6,10]$.

Perhaps the most widely used table of knots and links to appear in recent times is in Rolfsen's wonderful book, Knots and links [18]. This table, which includes knots up to ten crossings and links up to nine crossings, was taken from Conway's table and is accompanied by beautiful drawings of each link.

Conway's link table, as well as Caudron's, lists only unoriented links, and there are currently no existing tables or even accepted nomenclature for oriented links. In this paper we provide such a table, listing all oriented links up to nine crossings. We also list, in the case of alternating links, all possible minimal diagrams of the same link, up to orientation. As expected, these diagrams are all related by flypes. For nonalternating links we list only one diagram from each oriented link type because the number of different minimal diagrams that can represent a nonalternating link is often quite large. This is due in part to the possibility of changing the diagram by means of Type III Reidemeister moves. These moves cannot present themselves in alternating diagrams. Because of the recent resurgence of interest in combinatorial link invariants which can be defined starting with link diagrams we hope that this additional information will prove valuable. Moreover, because of recent applications of knot theory to chemistry and biochemistry, and because it is usually more natural to consider oriented links rather than unoriented links in such applications, we hope that these tables will prove useful to researchers in those fields. We also provide the values of the Alexander, HOMFLY, Jones, Kauffman, and BLM/Ho polynomials for each of the links.

Our table was generated "from scratch" rather than by introducing orientations into already existing tables. Thus, our work provides a check on Conway's table in the range in which they overlap. We are happy to report that we discovered no errors.

We assume that the reader is already familiar with the theory of knots and links as expounded, for example, in [18]. If $L$ is a link in $S^{3}$, we shall denote its reflection through an equator of $S^{3}$ by $L^{*}$. We call $L^{*}$ the enatiomorph or obverse of $L$. If additionally $L$ is oriented, then its reverse, $-L$, is obtained by reversing the orientations of all of its components. Finally the inverse, $-L^{*}$, is obtained by doing both. As is typical with previous tables, our table will list 
only one link from each set $\left\{L,-L, L^{*},-L^{*}\right\}$. In general, these four links need not be equivalent, that is, ambient isotopic. Furthermore, we list only prime nonsplit links.

Recall that the skein polynomials can never detect instances of $L \not \equiv-L$, and we avoid this issue altogether. On the other hand, the skein polynomials are quite good at detecting instances of $L \not L^{*}$. (If $L \cong L^{*}$, then $F_{L}(a, x)=F_{L}\left(a^{-1}, x\right)$ and $P_{L}(v, z)=P_{L}\left(-v^{-1}, z\right)$.) Indeed, within the range of this table, the Kauffman polynomial $F(a, x)$ detects all such cases. The only acheiral links (ones with $L \cong L^{*}$ ) are $6_{2}^{3}, 8_{4}^{3}, 8_{4}^{3}++-, 8_{6}^{3}, 8_{3}^{4}$, and $8_{3}^{4}+++-$. Interestingly, there are two cheiral links, $8_{2}^{3}++-$ and $8_{7}^{3}$, which are not detected by the HOMFLY polynomial $P(v, z)$. Finally, we point out that nearly all the links in this table are visibly arborescent (algebraic), so that their symmetries can be determined by the algorithm of Bonahon and Siebenmann [2].

One place where we may differ from accepted terminology is in our usage of the words projection and diagram. By a projection we shall mean a regular projection of a link where crossings appear as transverse doublepoints. By a diagram we shall mean a projection where over- and undercrossings have been indicated at each doublepoint, so that the link can be recovered from the diagram. Thus, associated with each projection with $n$ double points are $2^{n}$ possible diagrams.

Our general approach to tabulating links is to first list all possible projections and then pass from these to diagrams. Our methods are basically generalizations of those used by Dowker and Thistlethwaite for knots [5]. Our algorithms will work equally well for knots as for links.

This paper is an expanded version of the first author's Master's thesis written at Oregon State University in the spring of 1988.

\section{ENCODING AND LISTING LINK PROJECTIONS}

We use Dowker and Thistlethwaite's notation to encode a link projection. Consider the projection shown in Figure 1.1. If we first order the components, orient them, and choose a basepoint on each, we may then consecutively number the crossing points as we traverse the link subject to these choices. Each double point receives two integer labels. It is not hard to prove that given any choice of ordering and orientations of the components, some choice of basepoints will yield a numbering having both an even and an odd number at every double point. We shall only consider such parity reversing numberings.

Once the projection has been so labeled, there is an obvious involution $\alpha$ among the numbers $1,2, \ldots, 2 n$, where the projection has $n$ doublepoints. Namely, $\alpha(i)=j$ provided $i$ and $j$ label the same doublepoint. By listing only the even numbers $\alpha(1), \alpha(3), \ldots, \alpha(2 n-1)$, we may completely describe the involution $\alpha$. Moreover, we may position vertical bars within this list to indicate the presence of the various components. Thus, the projection of Figure 1.1 can be encoded by the sequence $610 \mid 82124$. 


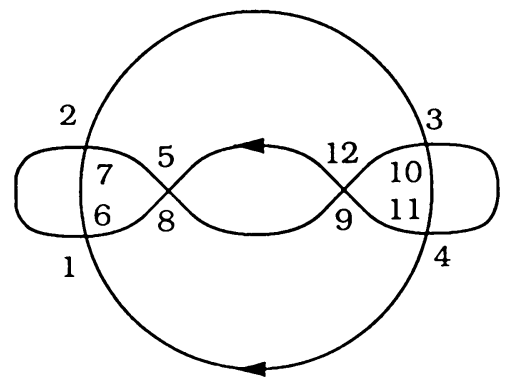

$610 \mid 82124$

FIGURE 1.1

We call the subsequences into which the sequence is partitioned by the bars the components of the sequence. The number of integers in each component is the length of that component. It is very useful to think of each component as being not only linearly ordered but also cyclically ordered. Thus, in the sequence $610 \mid 82124$ the 6 follows the 10 in the first component and the 8 follows the 4 in the second. It will also be convenient to define an "addition modulo components." If $i$ is used to label some crossing of the projection, then let $i+{ }_{c} k$ be the label obtained by starting at $i$ and advancing $k$ crossings on that component. Thus, in $610 \mid 82124$ we have $3+{ }_{c} 2=1$ while $8-{ }_{c} 5=11$.

Of course, choosing different orientations, basepoints, etc. will produce difference sequences for the same projection. This establishes an equivalence relation among sequences derived from link projections. We call a sequence reduced if the bars divide the sequence into components of nondecreasing length. In each equivalence class of sequences we may choose a unique representative, called the standard sequence, by taking the minimum with respect to lexicographic order of all reduced sequences in that class. (Note that all reduced sequences in one equivalence class have their bars in the same positions.)

For example, there are 256 possible ways to label the projection of Figure 1.1. But half of these are not parity reversing, and of the half that are, only half again, or 64 , yield reduced sequences. Since the projection has an obvious two-fold symmetry which reverses both orientations, only 32 distinct reduced sequences exist. Of these the smallest, or standard, sequence is $610 \mid 21248$. If we begin with an oriented projection and maintain that orientation, then we are only free to change basepoints and reorder the components. We then deal with an oriented equivalence class containing oriented reduced sequences, the smallest of which is the oriented standard sequence for that projection. The oriented standard sequence for the projection in Figure 1.1 (with the orientations as shown) is $610 \mid 41228$. This is not as small as the (unoriented) standard sequence.

It is obviously a simple matter to generate all reduced sequences of even integers with a given number of integers and bars. But which of these can actually be derived from projections? In particular, which correspond to projections of prime nonsplit links having a minimal number of crossings? (We are told by Thistlethwaite that Conway has dubbed 1-component sequences that do not arise from knot projections "knits." Bogus link sequences are "locks.") 
Three elementary observations present themselves immediately. The first is that there cannot be too many bars in a sequence. For it is not hard to prove that if $L$ is a prime nonsplit link with $n>3$ crossings, then $L$ has at most $n / 2$ components. Thus, for example, if $n=6$ we need only consider sequences having one or two bars. Secondly, if $L$ is a prime nonsplit link with $n>2$ crossings, then every sequence representing any projection of $L$ always has at least two integers in each component. Finally, suppose that $P$ is a projection of a prime nonsplit link $L$ having a minimal number of crossings. Then $P$ is connected, since $L$ is nonsplit. Moreover, because $P$ is minimal and $L$ is prime, there cannot be any circle in the plane that meets $P$ transversely in two points not on the same edge of $P$. To describe how this last condition manifests itself in the corresponding sequence, it is useful to define an $\operatorname{arc}$ of a sequence.

If $S$ is a sequence, then by an arc of $S$ we shall mean a set of consecutive integers which is a proper subset of some component of $S$. Keep in mind that by consecutive we mean with respect to the cyclic ordering of the component. Thus, $[12,4,8]$ is an arc of $610 \mid 82124$. Now suppose $P$ is a projection which is cut transversely by a circle in exactly two points not on the same edge of $P$. It is not hard to show that this condition is true if and only if, given any sequence $S$ encoding $P$, there exists a subset $I$ of $S$ consisting of a union of components together with a single nonempty arc of $S$ such that for all $i \in I$ we have $\alpha(i)+{ }_{c} 1 \in I$, or for all $i \in I$ we have $\alpha(i)-{ }_{c} 1 \in I$. For example, if $S$ is the sequence $68 \mid 12241410$ and $I=\{6,8,2,4\}$, then $\left\{\alpha(i)-{ }_{c} 1 \mid i \in I\right\}=\left\{1-{ }_{c} 1,3-{ }_{c} 1,7-{ }_{c} 1,9-{ }_{c} 1\right\}=\{4,2,6,8\}=I$. We shall denote this equality either by $\alpha(I){ }_{c} 1=I$ or $\alpha(I)-{ }_{c} 1=I$. To see that such a sequence corresponds to a projection which is met transversely by a circle in two points on different edges, it suffices to prove that exactly two edges connect the crossings of $I$ with those of $S-I$.

We summarize these results in the following theorem.

Theorem 1.1. A reduced sequence $S$ of $n$ integers and $k$ bars which is derived from a minimal projection of a prime nonsplit link $L$ must satisfy the following three conditions:

S1. If $n>3$, then $k+1 \leq n / 2$.

S2. If $n>2$, then each component of $S$ has length two or more.

S3. If $\alpha$ is the involution determined by $S$, then there does not exist a subset $I$ of $S$ consisting of a union of components together with a single nonempty arc of $S$ such that $\alpha(I)+{ }_{c} 1=I$ or $\alpha(I)-{ }_{c} 1=I$.

Given a reduced sequence $S$ which satisfies the three conditions just described, it still may not correspond to a minimal projection of a prime nonsplit link. And even if it does, it may not be the standard sequence for that projection. To further address this problem, we introduce an associated oriented graph $\Gamma(S)$. Suppose $S=\alpha(1) \alpha(3) \cdots \alpha(2 n-1)$ is a sequence having $k$ bars. Let $b_{1}, b_{2}, \ldots, b_{k}$ be the positions of the bars, where $b_{i}=2 j$ if the $i$ th bar 
lies between $\alpha(2 j-1)$ and $\alpha(2 j+1)$. It is convenient to introduce $b_{0}=0$ and $b_{k+1}=2 n$. We may now construct the oriented graph $\Gamma(S)$ by beginning with $k+1$ disjoint real intervals $I_{1}, I_{2}, \ldots, I_{k+1}$, where each $I_{j}=\left[b_{j-1}, b_{j}\right]$ (oriented as usual), and identifying integer points within these intervals as follows. First the endpoints of each interval are identified so as to produce $k+1$ disjoint circles. Then each $i$ in $I_{r}, b_{r-1}<i \leq b_{r}$, is identified with $\alpha(i)$ in $I_{s}$ if $b_{s-1}<\alpha(i) \leq b_{s}$. We regard this graph as endowed with slightly more structure than an arbitrary 4-valent graph. This is because each vertex was produced by joining together two pairs of "opposite" edges. Thus, the edges of $\Gamma(S)$ are cyclically ordered around each vertex. The graph $\Gamma(S)$ can obviously be embedded in $\mathbb{R}^{3}$ but of course not necessarily in $\mathbb{R}^{2}$.

By considering the graph $\Gamma(S)$ associated with a sequence $S$ we may extend the equivalence relation already defined for sequences derived from link projections to all sequences. Namely, two sequences are equivalent if their associated unoriented graphs are the same. Two sequences are oriented equivalent if their associated oriented graphs are the same. If we start with an arbitrary 4-valent graph $\gamma$ with cyclically oriented edges surrounding each vertex, then it is clear how to produce a sequence $S$ such that $\gamma=\Gamma(S)$. Simply choose a basepoint on each "component" of $\gamma$ and orient each "component." We may then traverse the "components" and consecutively number each vertex as we pass it. Clearly, each equivalence class of sequences is generated by renumbering the associated graph $\Gamma$ subject to different choices of basepoints, orientations, etc.

We call a sequence $S$ realizable if $\Gamma(S)$ can be embedded in $\mathbb{R}^{2}$ so that the cyclic order of the edges at each vertex is preserved in the obvious way. Thus, the realizable sequences are those that can be derived from link projections. If we view the embedding of $\Gamma(S)$ as lying in $S^{2}=\mathbb{R}^{2} \cup\{\infty\}$, then we may define two embeddings to be equivalent if one can be obtained from the other by composing with a homeomorphism of $S^{2}$. If a sequence $S$ is realizable, then it may be possible to embed $\Gamma(S)$ in $\mathbb{R}^{2}$ in different ways. However, if $S$ satisfies conditions S1-S3 of Theorem 1.1, then this is not possible. For it is not hard to prove that if $S$ satisfies conditions S1-S3, then $\Gamma(S)$ satisfies the following four conditions:

G1. The graph $\Gamma(S)$ is a 4-valent oriented graph with cyclically ordered edges surrounding each vertex.

G2. Every edge of $\Gamma(S)$ joins two distinct vertices.

G3. If any two edges of $\Gamma(S)$ are removed, then $\Gamma(S)$ remains connected.

G4. If any vertex of $\Gamma(S)$ is removed, then $\Gamma(S)$ remains connected.

It is now proven in [5, Lemma 1] that these conditions guarantee that any embedding of $\Gamma(S)$ in $\mathbb{R}^{2}$ is unique. This gives us the following important theorem.

Theorem 1.2. Unoriented minimal projections of prime nonsplit links, up to homeomorphism in $S^{2}$, are in one-to-one correspondence with realizable standard sequences satisfying conditions S1-S3. 


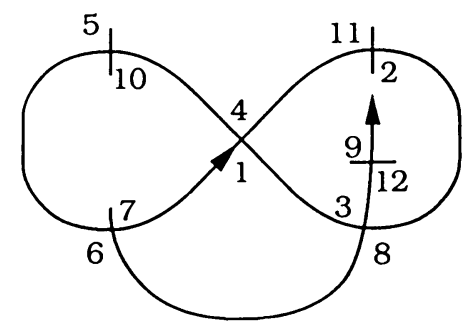

FIGURE 1.2

Thus, our task is to generate all possible reduced sequences satisfying $\mathrm{S} 1$ and S2 and then to eliminate those that do not satisfy S3, are not standard, or are not realizable. Only testing for realizability presents any real difficulty. For a given sequence $S$, how can we decide if it is realizable? A few examples will illustrate our algorithm. Consider first the sequence $S$ given by $4810 \mid 6122$. We may embed the first component and the beginning of the second component of $\Gamma(S)$ in $\mathbb{R}^{2}$ as shown in Figure 1.2. It is now necessary to connect the vertex $\{9,12\}$ to the vertex $\{5,10\}$. However this is impossible because the two vertices lie in different complementary regions of that portion of $\Gamma$ that has been embedded so far. In fact they are separated by the circle made up of the edges [1,2], [2, 3], and [3, 4]. It is more convenient to consider the circuit of edges $[9,8],[3,4],[1,2],[2,3],[8,9]$, which we call the obstruction circuit. Since the vertex $\{5,10\}$ is not present on the obstruction circuit, it is not possible to continue embedding the graph.

We formalize this idea as follows. First, given a sequence $S$ with $n$ entries and $k$ bars, let $b_{1}, b_{2}, \ldots, b_{k}$ be the positions of the bars as defined earlier. As before, let $b_{0}=0$ and $b_{k+1}=2 n$. Assume that for each $i$ we have $\alpha\left(b_{i}+1\right)<$ $b_{i}+1$. This implies that each component is connected to the previous ones. (This may not be true for a standard sequence.) For $i=1,2, \ldots, 2 n$, let $\gamma_{i}$ denote the subgraph of $\Gamma(S)$ corresponding to $I_{1} \cup I_{2} \cup \cdots \cup I_{r} \cup\left[b_{r}+1 / 2, i+1 / 2\right]$, where $b_{r}<i \leq b_{r+1}$. Hence, if $\eta$ is an embedding of $\Gamma(S)$, then $\eta\left(\gamma_{1}\right) \subset$ $\eta\left(\gamma_{2}\right) \subset \cdots \subset \eta\left(\gamma_{2 n}\right)$ are increasingly more complete embeddings of $\Gamma(S)$. Our strategy in attempting to embed $\Gamma(S)$ is to start with an embedding of $\gamma_{1}$ and extend it step by step through embeddings of the $\gamma_{i}$ until an embedding of $\gamma_{2 n}=\Gamma(S)$ is reached. Note that nothing can obstruct this process before the first loop is completed, i.e., before the first complete crossing is embedded. Also, embedding the first crossing of a new component, which is where it connects to a previously embedded component, presents no difficulty.

Obviously, $S$ is not realizable if and only if there exists an $i$ such that $\gamma_{i}$ can be embedded while $\gamma_{i+1}$ cannot. In particular, $S$ is not realizable if there exists an $i$ such that

(i) $\gamma_{i}$ can be embedded,

(ii) $\alpha(i+1)<i$, and

(iii) for all possible embeddings $\eta$ of $\gamma_{i}, \eta(\alpha(i+1))$ and $\eta(i)$ lie in different complementary regions of $\eta\left(\gamma_{i}\right)$. 
Assume that $\eta$ is an embedding of $\gamma_{i}$. The ith obstruction circuit $\mathscr{O}_{i}$ is the boundary of the region of $\mathbb{R}^{2}-\eta\left(\gamma_{i}\right)$ that contains $\eta(i+1 / 2)$. Clearly, we can extend the embedding of $\gamma_{i}$ to $\gamma_{i+1}$ if and only if $\eta(\alpha(i+1))$ lies on $\mathscr{O}_{i}$. Thus, we need to determine which integers lie on $\mathscr{O}_{i}$. To do this, we simply back up from $\eta(i+1 / 2)$ "turning right" at each crossing until we return to $\eta(i+1 / 2)$.

To facilitate this, it is convenient to imagine $\eta(i)$ not as a partial projection but rather as a partial diagram that furthermore is "descending." This means that at a crossing labeled $\{j, \alpha(j)\}$, we have $\eta(j)$ on the overcrossing strand precisely when $j<\alpha(j)$. As usual, we shall assign \pm 1 to each crossing where a crossing of type $X$ is assigned +1 while $X$ is assigned -1 . The sign of the first crossing to be embedded will always be (arbitrarily) chosen as +1 . If $S$ is realizable, then this uniquely determines the signs of the remaining crossings because the embedding is unique up to homeomorphism in $S^{2}$.

As we successfully embed $\gamma_{i}$ for larger and larger $i$, we record which crossings have been embedded and their signs. To determine $\mathscr{O}_{i}$, we begin by backing up from $\eta(i+1 / 2)$ until the first embedded crossing $\{\eta(j), \eta(\alpha(j))\}$ is reached. Initially, as we back up, we are travelling opposite to the orientation of $\gamma_{i}$. But later in our traversal of $\mathscr{O}_{i}$ we may be going with or against the orientation of $\gamma_{i}$. We must keep track of our current status in this regard. When we reach the crossing $\{\eta(j), \eta(\alpha(j))\}$, we know whether we have arrived at $\eta(j)$ or $\eta(\alpha(j))$. We can compare these two numbers and since the diagram is descending thus know if we have arrived on the over- or undercrossing strand. Suppose, for example, that we have arrived at $\eta(j)$, the crossing has sign $-1, j<\alpha(j)$ (so that we are on the overcrossing strand) and that we had been going forward along $\gamma_{i}$. Then to "turn right" at the crossing is to proceed from $\eta(\alpha(j))$ to $\eta(\alpha(j)+1)$ rather than to $\eta(\alpha(j)-1)$. Thus, it is not difficult to record $\mathscr{O}_{i}$.

Having determined $\mathscr{O}_{i}$, we can check if $\eta(\alpha(i+1))$ lies on $\mathscr{O}_{i}$. If so, then we may extend $\gamma_{i}$ to $\gamma_{i+1}$ and record the sign of the newly embedded crossing. If $\gamma_{i}$ can be extended to $\gamma_{i+1}$ in only one way, then the sign of the new crossing is easily determined by comparing the orientations of $\mathscr{O}_{i}$ and $\eta\left(\gamma_{i}\right)$ at $\eta(\alpha(i+1))$. However, there may be two ways to extend $\gamma_{i}$ to $\gamma_{i+1}$. If so, we choose one way and continue with the algorithm. If we fail to embed some future $\gamma_{k}$, then we must return to this step, choose differently, and continue again. The sequence $612 \mid 210144168$ illustrates this possibility. After $\gamma_{9}$ has been embedded, $\gamma_{10}$ may be embedded with the crossing $\{7,10\}$ having a sign of either \pm 1 . If -1 is chosen, then $\gamma_{14}$ cannot be embedded. But if +1 is chosen, then the embedding can be extended all the way to $\Gamma=\gamma_{16}$.

Using a Macintosh SE computer, programs were written in PASCAL to implement the algorithms described above. For each $n=1,2, \ldots, 9$, all possible sequences satisfying conditions S1 and S2 were generated in lexicographic order. As each new sequence was generated, it was first tested to see if it satisfied condition S3. If so, it was then tested to see if it was standard. This was done by generating all other equivalent sequences and stopping if a smaller one was reached. The equivalent sequences were generated by methodically relabeling the associated graph $\Gamma(S)$. What remained was a list of all standard sequences 
satisfying conditions S1-S3. These standard sequences were then tested for realizability, finally giving rise to a list of all possible unoriented projections.

Since testing a sequence for realizability is very time consuming and does not depend on orientations, we first generated all unoriented projections. The list of oriented projections was then derived from this by considering all possible orientations for a given projection and determining the oriented standard sequences within each unoriented equivalence class.

Another method for testing a sequence for realizability, which may be much faster than ours, has been pointed out by the referee. This method reduces the problem to that of testing a knot sequence for realizability and in that case uses the algorithm of Dowker and Thistlethwaite [5]. Suppose $S$ is a sequence with $k$ crossings and $n$ components. Then we may create a new sequence $S^{\prime}$ with $k+1$ crossings and $n-1$ components by formally replacing a crossing $X$ belonging to two different components with either $\gamma$ or $\varnothing$. Then $S$ is realizable if and only if $S^{\prime}$ is.

\section{PASSING FROM PROJECTION TO DIAGRAMS}

Our goal, of course, is not to enumerate link projections but rather link diagrams and consequently links themselves. Therefore, we must now pass from the list of projections we have generated to one of diagrams. Each oriented projection with $n$ doublepoints determines $2^{n}$ possible oriented diagrams. These diagrams come in pairs with each pair representing a link $L$ and its obverse $L^{*}$. Since we only intend to list one link from each set $\left\{L, L^{*},-L,-L^{*}\right\}$, we may eliminate half of the diagrams. The situation is particularly simple if we restrict our attention to alternating links. In this case we need only consider the single alternating diagram obtained by placing each odd label on the overcrossing strand and each even label on the undercrossing strand. Thus, we may consider each sequence as encoding not an oriented projection but rather an oriented alternating diagram.

To encode nonalternating diagrams, we may modify the sequence corresponding to the associated projection in a simple way. Consider the nonalternating diagram shown in Figure 2.1. The associated projection can be encoded by $610 \mid 842$. The alternating diagram produced from this by placing the odd labels on the overpasses differs from the nonalternating diagram at two crossings: $\{2,9\}$ and $\{3,10\}$. We may note this by introducing minus signs into the sequence, thus obtaining the signed sequence $6-10 \mid 84-2$.

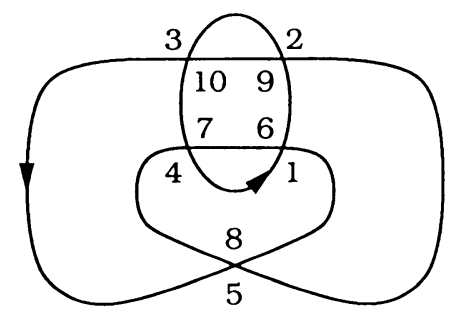

$610 \mid 842$

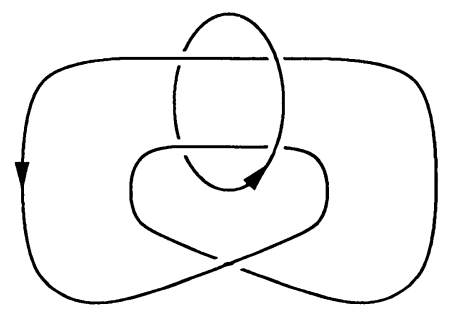

$6-10 \mid 84-2$

FIGURE 2.1 
Again, since we only wish to list one link from $\left\{L, L^{*},-L,-L^{*}\right\}$, we need only consider signed sequences which begin with a positive integer. Of course, many of these signed sequences encode nonalternating diagrams which are not minimal or which represent split or nonprime links. Most of these can easily be detected and discarded by the computer.

Finally, what remains is a list of realizable sequences which encode the alternating diagrams and a list of signed sequences derived from these which encode the nonalternating diagrams.

\section{PASSING FROM DIAGRAMS TO LINKS}

The list of sequences and signed sequences generated thus far is complete, containing every possible minimal diagram of an oriented prime nonsplit link having a given number of crossings or less. (In our case, nine crossings.) However the list contains many repetitions in the sense that several diagrams can represent the same link.

In order to distinguish diagrams representing different links from each other we computed the HOMFLY and Kauffman polynomials of each. These polynomials, as well as the Alexander, Jones, and BLM/Ho polynomials which can be derived from them, are listed in Appendix $\mathrm{C}$ on the microfiche supplement attached to the inside cover of this issue. Fortunately, we did not need to compare two alternating diagrams having different numbers of crossings. For it was conjectured by Tait, and recently proven by Kauffman, Murasugi, and Thistlethwaite, that all minimal alternating diagrams of a prime alternating link have the same number of crossings $[7,14,21]$.

In all but nine cases these polynomial invariants sufficed to distinguish different links. Otherwise, links having the same polynomials were shown to be the same, in fact related by flypes in the case of alternating links. The nine sets of links not distinguished by their polynomial invariants are
(1) $8_{1}^{4}+++-8_{1}^{4}++--$
(4) $9_{4}^{3}++-9_{4}^{3}+-+$
(2) $8_{3}^{4}$
$8_{3}^{4}+++-$
(3) $9_{3}^{3}$
$9_{3}^{3}+-+$
(5) $9_{12}^{3}$
$9_{12}^{3}+-+$
(6) $9_{15}^{3}++-9_{15}^{3}+-+$
(7) $9_{16}^{3} 9_{16}^{3}+--$
(8) $9_{17}^{3} 9_{17}^{3}+-+$
(9) $9_{21}^{3} 9_{21}^{3}+-+$.

Rather than listing these links by their standard sequences, we have given their "Rolfsen numbers." We describe this method of enumeration in the next section.

It is worth noting that in each of these pairs the two links are related by mutation, thus explaining the equality of their skein polynomials.

All cases except (5) and (9) are easily analyzed by considering the linking numbers of the various components of the links. These two cases, however, are more difficult. Since the links are arborescent, they can be distinguished using the algorithm of Bonahon and Siebenmann [2]. But since their work is still unpublished, we shall include for completeness sake an ad hoc argument that the two links in case (5) are indeed different. A similar argument works for case (9). We thank Francis Bonahon for suggesting this proof to us. 
The two links are pictured in Appendix A of the microfiche supplement. Let $L$ be $9_{12}^{3}$ and let $I, J$, and $K$ denote the first, second, and third components respectively. (Remember that the sequence is associated with a specific ordering of the components.) The second link, $9_{12}^{3}+-+$, is obtained from $L$ by reversing $J$. We thus seek to prove that $L=\{I, J, K\}$ is not equivalent to $L^{\prime}=\{I,-J, K\}$. Note that $\{I, K\}$ forms a Whitehead link, while $\{I, J\}$ and $\{J, K\}$ are both unlinks. Hence, if $\varphi$ is a homeomorphism of $S^{3}$ taking $L$ to $L^{\prime}$, then $\varphi$ must take $J$ to $-J$ and $\{I, K\}$ to $\{I, K\}$. In fact, $\varphi$ must take $I$ to $I$ and $K$ to $K$, since $\{D(I), J, K\}$ and $\{I,-J, D(K)\}$ have different HOMFLY polynomials, where $D(P)$ denotes the untwisted double of $P$. It now follows that $\varphi$ must be isotopic to the identity. Hence, there exists $\alpha \in \pi_{1}\left(S^{3}-\{I, K\}\right)$ such that $\alpha J \alpha^{-1}=J^{-1}$.

But the Whitehead link is hyperbolic, so that $\pi_{1}\left(S^{3}-\{I, K\}\right) \cong \Gamma$, a discrete subgroup of $\operatorname{Isom}\left(\mathbb{H}^{3}\right)$ acting freely on $\mathbb{H}^{3}$. Suppose that $J \neq 1$ and that $J$ fixes two ideal points, $x$ and $y$. Now $J \alpha(x)=J \alpha J(x)=\alpha(x)$, and so $\alpha(x)$ is also fixed by $J$. Thus, either $\alpha(x)=x$ and $\alpha(y)=y$, or $\alpha(x)=y$ and $\alpha(y)=x$. But the latter case is impossible, for $\alpha$ would then fix some point lying on the geodesic joining $x$ to $y$. It now follows that $\alpha$ commutes with $J$, since both are in $\operatorname{stab}\{x, y\}$ which is abelian. If $J$ fixes only one ideal point $x$, then again $x$ is also fixed by $\alpha$. Moreover, it is the only fixed point of $\alpha$, since otherwise, $\alpha$ and $J$ would generate a nondiscrete subgroup of $\operatorname{Isom}\left(\mathbb{H}^{3}\right)$. Hence, once again, we may conclude that $\alpha$ commutes with $J$.

TABLE 3.1

\begin{tabular}{crrrrrr}
\hline & & standard & unoriented & unoriented & oriented \\
crossings & sequences $^{1}$ & sequences & projections & links & links \\
\hline 1 & 0 & 0 & 0 & 0 & 0 \\
2 & 2 & 1 & 1 & 1 & 1 \\
3 & 0 & 0 & 0 & 0 & 0 \\
4 & 24 & 1 & 1 & 1 & 2 \\
5 & 120 & 1 & 1 & 1 & 1 \\
6 & 2,160 & 11 & 6 & 6 & 10 \\
7 & 15,120 & 29 & 8 & 9 & 14 \\
8 & 241,920 & 210 & 35 & 29 & 62 \\
9 & $2,540,160$ & 1462 & 97 & 83 & 156 \\
\hline
\end{tabular}

${ }^{1}$ Reduced sequences which satisfy conditions $\mathrm{S} 1$ and $\mathrm{S} 2$. 
Thus, $J^{2}=1$, and so $J=1$. But $J$ cannot be nullhomotopic in $S^{3}-$ $\{I, K\}$, as can be seen by examining the infinite cyclic cover of $S^{3}$ over $K$. For in this cover, there are lifts of $J$ which have nonzero linking with lifts of $I$. Thus, $L$ cannot be equivalent to $L^{\prime}$.

It is interesting to note that there is only one pair of links, $9_{12}^{2}$ and $9_{34}^{3}+-$, which can be distinguished by the Kauffman polynomial but not by the HOMFLY polynomial.

Finally, in Table 3.1 we summarize the numbers of sequences, projections, and diagrams that were generated for each crossing number $n$.

\section{How TO READ THE TABLES}

A table of oriented link diagrams of links with nine or fewer crossings is given in Appendix A of the microfiche supplement. Ignoring orientations, we have listed all possible minimal diagrams for each alternating link, and one minimal diagram for each nonalternating link. To list diagrams for each oriented link type would be too repetitive, since within each unoriented class, diagrams for different oriented links differ only by orientation. Thus, we show oriented diagrams for just one oriented link type within each unoriented class. Moreover, even within one oriented link type, we have not shown all possible minimal oriented diagrams, but rather only those which differ as unoriented diagrams. As we will soon explain, diagrams for the other oriented links are easily obtained from these.

While the lexicographic ordering of sequences provides a natural way to order the links within the table, we have kept with tradition and ordered them as in Conway's or Rolfsen's table. Furthermore, we have extended Rolfsen's notation. An example should serve to illustrate our schema.

Consider the unoriented link $9_{7}^{3}$. This is the seventh nine-crossing threecomponent link to appear in Conway's or Rolfsen's table. The smallest sequence representing this link, taken over all possible orientations and over all possible diagrams, is $4814|21216| 61810$. The oriented alternating diagram determined by this sequence is pictured first in Appendix A. (We have chosen to draw all the diagrams in the table with the odd-labeled doublepoints as overcrossings and the first crossing as positive. This convention sometimes results in different diagrams for the same oriented link differing not only by flypes, but also by reflection.) We denote this oriented link by $9_{7}^{3}+++$ or more simply as $9_{7}^{3}$. The sequence actually corresponds to an ordering of the components, and we have indicated this by labeling the components 1,2 , and 3 . We have also placed the arrowheads at the locations of the basepoints corresponding to the sequence. Oriented links obtained from $9_{7}^{3}+++$ by reversing orientations of various components will be denoted by $9_{7}^{3}++-, 9_{7}^{3}+-+, 9_{7}^{3}+--$, etc., where a "-" in the $i$ th position indicates that the orientation of the $i$ th component has been reversed. Since we do not distinguish a link from its reverse, there are 
only four possibilities instead of eight, and all our link names will begin with "+". In the case of $9_{7}^{3}$, however, only $9_{7}^{3}+++$ and $9_{7}^{3}++-$ are distinct. The fact that there are two oriented link types is indicated in the table by the two in parentheses which follows $9_{7}^{3}$. Thus, a diagram for $9_{7}^{3}++-$ can be obtained from the pictured one by reversing the component labeled 3. To save space, we have not drawn this diagram but we have given its oriented standard sequence $4814|21218| 16610$, as well as the oriented standard sequence for $9_{7}^{3}+++$, in Appendix B. Thus, by looking in Appendix B, one can learn which components to reverse in the pictured diagram so as to produce the other oriented links in a given unoriented class.

There are two more unoriented diagrams that represent the unoriented link $97_{7}^{3}$. Standard sequences for these unoriented diagrams are given in Appendix B and are $610|214| 41816812$ and $612|21016| 418814$. The two oriented diagrams that these sequences determine are pictured next. They are of the same oriented link type as $9_{7}^{3}+++$. But each of these two diagrams may be reoriented so as to represent $9_{7}^{3}++-$. Oriented standard sequences for these oriented diagrams are $610|414| 21816812$ and $612|41016| 218814$ and again, these sequences are given in Appendix B.

Nonalternating links generally have too many unequivalent diagrams for us to list here. Thus, for each nonalternating oriented link type we give only one diagram. This diagram corresponds to a signed sequence which is minimal when all minus signs are removed. If more than one such signed sequence exists, then we have chosen one beginning with a positive integer and having the fewest minus signs. Thus, each nonalternating diagram "sits over" the smallest possible (with respect to lexicographic order) alternating diagram.

Finally, in Appendix C we list the HOMFLY, Kauffman, Jones, BLM/Ho, and Alexander polynomials of each link. The Alexander polynomial is given in the normalized form due to Conway.

We have adopted the following form of the skein relations for these polynomials. The reader is warned that these may differ from conventions used by other authors. Suppose $D_{+}, D_{-}$, and $D_{0}$ are three oriented diagrams that are identical except near a single crossing of $D_{+}$, where they differ as shown in Figure 4.1(a).

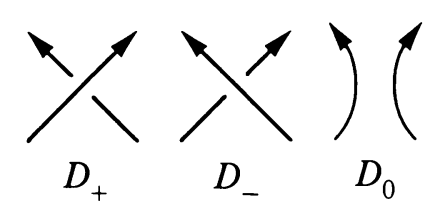

(a)

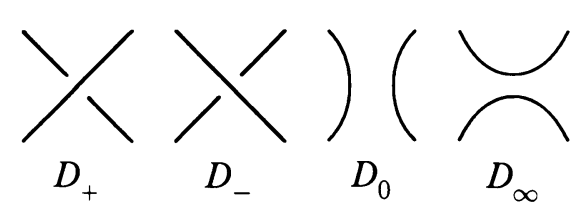

(b)

FIGURE 4.1 
Then the HOMFLY polynomial satisfies

$$
P: \quad v^{-1} P_{D_{+}}-v P_{D_{-}}=z P_{D_{0}} \text { and } P(\text { unknot })=1 .
$$

The Kauffman polynomial of an oriented link $L$ is defined by

$$
F: \quad F_{L}(a, x)=a^{-w(D)} \Lambda(|D|),
$$

where $D$ is any oriented diagram of $L, w(D)$ is the writhe of $D,|D|$ is $D$ stripped of its orientation, and $\Lambda$ is the regular isotopy invariant of unoriented diagrams given by

$$
\begin{array}{ll} 
& \text { (i) } \Lambda(0)=1, \\
\Lambda: & \text { (ii) } \Lambda(\mathbb{D} \sim)=a \Lambda(D), \Lambda(\mathbb{D})=a^{-1} \Lambda(D), \\
& \text { (iii) } \Lambda\left(D_{+}\right)+\Lambda\left(D_{-}\right)=x\left(\Lambda\left(D_{0}\right)+\Lambda\left(D_{\infty}\right),\right.
\end{array}
$$

where $D_{+}, D_{-}, D_{0}$, and $D_{\infty}$ are four unoriented diagrams which are identical except near a single crossing of $D_{+}$, where they differ as shown in Figure 4.1(b). Moreover, the Jones, BLM/Ho, and Conway polynomials are given by

$$
V(t)=P\left(t, t^{1 / 2}-t^{-1 / 2}\right), \quad Q(x)=F(1, x), \quad \text { and } \quad \nabla(z)=P(1, z) .
$$

To abbreviate these polynomials, we use a notation which is now standard. (See, for example, [10].) A single example should suffice to explain the system.

The entry for $7_{1}^{3}+--$ appears as follows.

$$
\begin{aligned}
7_{1}^{3}+-- & \{-2\}([0] 01-21)([0] 03-3)([0] 031-1)([0] 011) \\
& \{-2\}(-1-2-10[0])(2200 *)(3530[0])(-3-300 *) \\
& (-3-5-5-30[0])(1-3-4000 *) \\
& (33110[0])(34100 *)(1100[0]) \\
& \{4 / 2\}(1-14-34-33-1) \\
& \{-2\}(-4411-6-16-6882) \\
& \{-2\}(0032)
\end{aligned}
$$

This means that

$$
\begin{aligned}
& \begin{aligned}
& P(v, z)=z^{-2}\left[\left(v^{4}-2 v^{6}+v^{8}\right)\right.+\left(3 v^{4}-3 v^{6}\right) z^{2} \\
&\left.+\left(3 v^{4}+v^{6}-v^{8}\right) z^{4}+\left(v^{4}+v^{6}\right) z^{6}\right], \\
& \begin{aligned}
F(a, x)=x^{-2}[( & \left.-a^{-8}-2 a^{-6}-a^{-4}\right)+\left(2 a^{-7}+2 a^{-5}\right) x \\
& +\left(3 a^{-8}+5 a^{-6}+3 a^{-4}\right) x^{2}+\left(-3 a^{-7}-3 a^{-5}\right) x^{3} \\
& +\left(-3 a^{-10}-5 a^{-8}-5 a^{-6}-3 a^{-4}\right) x^{4} \\
& +\left(a^{-11}-3 a^{-9}-4 a^{-7}\right) x^{5}+\left(3 a^{-10}+3 a^{-8}+a^{-6}+a^{-4}\right) x^{6} \\
& \left.\quad+\left(3 a^{-9}+4 a^{-7}+a^{-5}\right) x^{7}+\left(a^{-8}+a^{-6}\right) x^{8}\right],
\end{aligned} \\
& V(t)=t^{2}\left(1-t+4 t^{2}-3 t^{3}+4 t^{4}-3 t^{5}+3 t^{6}-t^{7}\right), \\
& Q(x)=x^{-2}\left(-4+4 x+11 x^{2}-6 x^{3}-16 x^{4}-6 x^{5}+8 x^{6}+8 x^{7}+2 x^{8}\right), \\
& \nabla(z)= z^{-2}\left(3 z^{4}+2 z^{6}\right) .
\end{aligned}
\end{aligned}
$$




\section{BIBLIOGRAPHY}

1. J. W. Alexander and G. B. Briggs, On types of knotted curves, Ann. of Math. 28 (1926-27), 562-586.

2. F. Bonahon and L. Siebenmann, Geometric splittings of classical knots and the algebraic knots of Conway, preprint.

3. A. Caudron, Classification des noeuds et des enlacements, Prépublications Univ. Paris-Sud, Orsay, 1981.

4. J. H. Conway, An enumeration of knots and links and some of their related properties, Computational Problems in Abstract Algebra (John Leech, ed.), Pergamon Press, Oxford and New York, 1969, pp. 329-358.

5. C. H. Dowker and M. B. Thistlethwaite, Classification of knot projections, Topology Appl. 16 (1983), 19-31.

6. L. Kauffman, New invariants in the theory of knots, Amer. Math. Monthly 95 (1987), 395407.

7. __ State models and the Jones polynomial, Topology 26 (1987), 395-407.

8. T. P. Kirkman, The 364 unifilar knots of ten crossings enumerated and defined, Trans. Roy. Soc. Edinburgh 32 (1885), 483-506.

9. - The enumeration, description and construction of knots of fewer than ten crossings, Trans. Roy. Soc. Edinburgh 32 (1885), 281-309.

10. W. B. R. Lickorish and K. Millett, The new polynomial invariants of knots and links, Math. Mag. 61 (1988), 3-23.

11. C. N. Little, On knots with a census for order ten, Trans. Connecticut Acad. 7 (1885), 27-43.

12. __ Alternate \pm knots of order 11, Trans. Roy. Soc. Edinburgh 36 (1890), 253-255.

13. __ Nonalternate \pm knots, Trans. Roy. Soc. Edinburgh 39 (1900), 771-778.

14. K. Murasugi, Jones polynomials and classical conjectures in knot theory, Topology 26 (1987), 187-194.

15. K. A. Perko, Jr., On the classification of knots, Proc. Amer. Math. Soc. 45 (1974), 262-266.

16. __ On 10-crossing knots, Portugal. Math. 38 (1979), 5-9.

17. K. Reidemeister, Knotentheorie, Ergeb. Math. Grenzgeb., Bd. 1, Springer-Verlag, Berlin, 1932.

18. D. Rolfsen, Knots and links, Publish or Perish, 1976.

19. P. G. Tait, On knots. I, II, III, Scientific Papers, Vol. I, Cambridge Univ. Press, London, 1898, pp. 273-347.

20. M. B. Thistlethwaite, Knot tabulation and related topics, Aspects of Topology (I. M. James and E. H. Kronheimer, eds.), London Math. Soc. Lecture Notes Ser., vol. 93, Cambridge Univ. Press, 1985, pp. 1-76.

21. __ A spanning tree expansion of the Jones polynomial, Topology 26 (1987), 297-309.

Department of Mathematics, University of California at Santa Barbara, Santa BarBARA, CALIFORNIA 93106

Department of Mathematics, Pitzer College, Claremont, California 91711

E-mail address: jhoste@pitzer.claremont.edu 